Bull. Korean Math. Soc. 51 (2014), No. 2, pp. 501-510

http://dx.doi.org/10.4134/BKMS.2014.51.2.501

\title{
CHARACTERIZATIONS AND THE MOORE-PENROSE INVERSE OF HYPERGENERALIZED K-PROJECTORS
}

\author{
MARINA TOŠIĆ
}

\begin{abstract}
We characterize hypergeneralized k-projectors (i.e., $A^{k}=$ $\left.A^{\dagger}\right)$. Also, some representation for the Moore-Penrose inverse of a linear combination of hypergeneralized k-projectors is found and the invertibility for some linear combinations of commuting hypergeneralized $\mathrm{k}$-projectors is considered.
\end{abstract}

\section{Introduction}

Let $\mathbb{C}^{n \times m}$ denote the set of all $n \times m$ complex matrices. The symbols $\mathcal{R}(A)$ and $\mathrm{r}(A)$ will denote the range (column space) and the rank of a matrix $A$, respectively. For a matrix $A \in \mathbb{C}^{n \times n}, \operatorname{tr}(A)$ and $\sigma(A)$ will denote the trace and the spectrum of a matrix $A$, respectively. Also, we will use the following notation: for $k \in \mathbb{N}$ and $k>1$, the set of complex roots of 1 shall be denoted by $\sigma_{k}$ and if we set $\omega_{k}=e^{2 \pi i / k}$, then $\sigma_{k}=\left\{\omega_{k}^{0}, \omega_{k}^{1}, \ldots, \omega_{k}^{k-1}\right\}$. The Moore-Penrose inverse of $A$ is the unique matrix $A^{\dagger}$ satisfying the equations
(1) $A A^{\dagger} A=A$,
(2) $A^{\dagger} A A^{\dagger}=A^{\dagger}$,
(3) $A A^{\dagger}=\left(A A^{\dagger}\right)^{*}$,
(4) $A^{\dagger} A=\left(A^{\dagger} A\right)^{*}$.

$I_{n}$ will denote the identity matrix of order $n$ and $0_{r}$ will denote the nullmatrix of order $r$. Also, $P_{S}$ denotes the orthogonal projector onto subspace $S$. We use the notations $C_{n}^{P}, C_{n}^{O P}, C_{n}^{E P}, C_{n}^{G P}$ and $C_{n}^{H G P}$ for the subsets of $\mathbb{C}^{n \times n}$ consisting of projectors (idempotent matrices), orthogonal projectors (Hermitian idempotent matrices), EP (range-Hermitian) matrices, generalized and hypergeneralized projectors, respectively, i.e.,

$$
\begin{aligned}
& C_{n}^{P}=\left\{A \in \mathbb{C}^{n \times n}: A^{2}=A\right\}, \\
& C_{n}^{O P}=\left\{A \in \mathbb{C}^{n \times n}: A^{2}=A=A^{*}\right\}, \\
& C_{n}^{E P}=\left\{A \in \mathbb{C}^{n \times n}: \mathcal{R}(A)=\mathcal{R}\left(A^{*}\right)\right\}=\left\{A \in \mathbb{C}^{n \times n}: A A^{\dagger}=A^{\dagger} A\right\}, \\
& C_{n}^{G P}=\left\{A \in \mathbb{C}^{n \times n}: A^{2}=A^{*}\right\}, \\
& C_{n}^{H G P}=\left\{A \in \mathbb{C}^{n \times n}: A^{2}=A^{\dagger}\right\} .
\end{aligned}
$$

Received December 12, 2012; Revised September 14, 2013.

2010 Mathematics Subject Classification. 15A09.

Key words and phrases. hypergeneralized k-projector, linear combination, the MoorePenrose inverse, nonsingularity. 
Further, we will use the abbreviations "g-p" and "hg-p" for generalized projector and hypergeneralized projector, respectively.

A matrix $B \in \mathbb{C}^{n \times n}$ is said to be similar to a matrix $A \in \mathbb{C}^{n \times n}$ if there exists a nonsingular matrix $P \in \mathbb{C}^{n \times n}$ such that $B=P^{-1} A P$. If a matrix $A \in \mathbb{C}^{n \times n}$ is similar to a diagonal matrix, then $A$ is said to be diagonalizable.

The concepts of g-p and hg-p were introduced by Groß and Trenkler [11] who presented very interesting properties of the classes of g-p and hg-p. A characterization of nonnegative matrices such that $A=A^{\dagger}$ is derived by Berman $[7]$.

In [5], the authors introduced the following concept: A square matrix $A$ is said to be a k-generalized projector (g-kp) if $A^{k}=A^{*}$. This class of matrices obviously generalizes to the class of g-p. In [9], the g-kp have been generalized on the set of all bounded linear operators on Hilbert space. They defined the hypergeneralized k-projectors (hg-kp): Let $\mathcal{H}$ be a Hilbert space and $\mathcal{B}(\mathcal{H})$ the all bounded linear operators on $\mathcal{H}$. For $A \in \mathcal{B}(\mathcal{H}), A$ is said to be a hypergeneralized k-projector if there exists a natural number $k>1$ such that $A^{k}=A^{\dagger}$. Also, they proved the following inclusion: The set of all g-kp is the subset of all hg-kp. Hence, the class of g-kp may be generalized by considering the class of hg-kp. This leads our interest to the subset of the class of square matrices $A$ with the property $A^{k}=A^{\dagger}$ for $k \in \mathbb{N}$ and $k>1$, called as hypergeneralized k-projectors. Specially, if $k=2$, we get the class of h-p (see [1], [2], [3], [11], [13], [14]).

In this paper, we characterize this class of matrices and, as simple corollaries, we deduce the characterizations of hg-p presented in [2] and [3]. Also, we give the form for the Moore-Penrose inverse and study the nonsingularity of a linear combination $c_{1} A+c_{2} B$, where $A$ and $B$ are commuting hg-kp, as well as the nonsingularity of a linear combination $c_{1} A+c_{2} B+c_{3} C$, where $A, B$ and $C$ are commuting hg-kp such that $B C=0$. Also, as corollaries for commuting g-p and hg-p, we give results presented in [14].

\section{Characterizations of hypergeneralized k-projectors}

In this section, we give some characterizations of hg-kp. First, we give necessary and sufficient conditions that $A$ is a hg-kp.

Theorem 2.1. Let $A \in \mathbb{C}^{n \times n}$ and $k \in \mathbb{N}, k>1$. Then the following statements are equivalent:

(i) $A$ is a $h g-k p$ (i.e., $\left.A^{k}=A^{\dagger}\right)$;

(ii) $A$ is a EP matrix, $\sigma(A) \subseteq \sigma_{k+1} \cup\{0\}$ and $A$ is diagonalizable;

(iii) $A$ is a EP matrix and $A^{\overline{k+2}}=A$.

Proof. Let us prove that (i) is equivalent to (iii).

(i) $\Rightarrow$ (iii) Matrix $A$ is EP because $A A^{\dagger}=A A^{k}=A^{k} A=A^{\dagger} A$. Also, matrix $A$ is $(k+2)$-potent because $A^{k+2}=A A^{k} A=A A^{\dagger} A=A$. 
(iii) $\Rightarrow$ (i) Since $A$ is an EP matrix, there exist a unitary matrix $U \in \mathbb{C}^{n \times n}$ and a nonsingular matrix $K \in \mathbb{C}^{r \times r}$ such that

$$
A=U(K \oplus 0) U^{*}
$$

(see [8]). Also,

$$
A^{\dagger}=U\left(K^{-1} \oplus 0\right) U^{*} .
$$

From $A^{k+2}=A$, we have $K^{k}=K^{-1}$, which implies that $A$ is a hg-kp. Hence, (i) holds.

(ii) $\Leftrightarrow$ (iii) This follows from the well known fact that $A^{k+2}=A$ if and only if $A$ is diagonalizable and the spectrum of $A$ is contained in $\sigma_{k+1} \cup\{0\}$ (see [6, Theorem 2.1]).

From Theorem 2.1, it follows that $A$ is a hg-kp if and only if

$$
A=U(K \oplus 0) U^{*},
$$

where $U \in \mathbb{C}^{n \times n}$ is a unitary matrix and $K \in \mathbb{C}^{r \times r}$ is a nonsingular matrix such that $K^{k+1}=I_{r}$.

If $A$ is a hg-kp, then $A^{k+1}=A A^{\dagger}$, i.e., $A^{k+1}$ is the orthogonal projector onto $\mathcal{R}(A)$. Also, the converse implication is valid.

Theorem 2.2. Let $A \in \mathbb{C}^{n \times n}$. Then $A$ is a hg-kp if and only if $A^{k+1}$ is the orthogonal projector onto $\mathcal{R}(A)$.

Proof. $(\Leftarrow)$ By Corollary 6 in $[12]$, every matrix $A \in \mathbb{C}^{n \times n}$ of rank $r$ has the form

$$
A=U\left[\begin{array}{cc}
D K & D L \\
0 & 0
\end{array}\right] U^{*}
$$

where $U \in C^{n \times n}$ is unitary, $D=\operatorname{diag}\left(\lambda_{1} I_{r_{1}}, \ldots, \lambda_{t} I_{r_{t}}\right)$ is the diagonal matrix of nonzero singular values of $A, \lambda_{1}>\lambda_{2}>\cdots>\lambda_{t}>0, r_{1}+r_{2}+\cdots+r_{t}=r$ and $K \in \mathbb{C}^{r \times r}, L \in \mathbb{C}^{r \times(n-r)}$ satisfy

$$
K K^{*}+L L^{*}=I_{r} .
$$

From (4), it follows that

$$
A^{k+1}=U\left[\begin{array}{cc}
(D K)^{k+1} & (D K)^{k} D L \\
0 & 0
\end{array}\right] U^{*}
$$

and

Hence,

$$
A^{\dagger}=U\left[\begin{array}{cc}
K^{*} D^{-1} & 0 \\
L^{*} D^{-1} & 0
\end{array}\right] U^{*}
$$

$$
A A^{\dagger}=P_{\mathcal{R}(A)}=U\left(I_{r} \oplus 0\right) U^{*} .
$$

Now, $P_{\mathcal{R}(A)}=A^{k+1}$ if and only if $(D K)^{k+1}=I_{r}$ and $L=0$. Thus, $A$ has the form (3), which is equivalent to the fact that $A$ is a hg-kp.

Corollary 2.3. Let $A \in \mathbb{C}^{n \times n}$ be a hg-kp. Then $\mathrm{r}(A)=\operatorname{tr}\left(A^{k+1}\right)$. 
Proof. From Theorem 2.2 and (3) we get $\mathrm{r}(A)=\mathrm{r}\left(A^{k+1}\right)=\operatorname{tr}\left(A^{k+1}\right)$.

The converse result is invalid, as can be seen by taking

$$
A=\left[\begin{array}{ll}
1 & 1 \\
0 & 1
\end{array}\right]
$$

in which case $\mathrm{r}(A)=\operatorname{tr}\left(A^{k}\right)$ and $A^{\dagger}=A^{-1} \neq A^{k}$ for $k \in \mathbb{N}$. Hence, $A$ is not a hg-kp.

As corollaries, we get Theorem 1 and Corollary 1 in [3].

Corollary $2.4([3])$. Let $A \in \mathbb{C}^{n \times n}$. Then $A \in C_{n}^{H G P}$ if and only if $A^{3}$ is the orthogonal projector onto $\mathcal{R}(A)$.

Corollary 2.5 ([3]). Let $A \in C_{n}^{H G P}$. Then $r(A)=\operatorname{tr}\left(A^{3}\right)$.

By definition of the Moore-Penrose inverse, the group inverse and the Drazin inverse, it is easy to see that if $A$ is a hg-kp, then

$$
A^{\dagger}=A^{\sharp}=A^{d}=A^{k}=A^{m(k+1)+k}, \quad m \in \mathbb{N} .
$$

Generally $A$ is a hg-kp if and only if its Moore-Penrose inverse $A^{\dagger}$ is:

Theorem 2.6. Let $A \in \mathbb{C}^{n \times n}$. The following are equivalent:

(i) $A$ is a hg-kp;

(ii) $A^{*}$ is a $h g-k p$;

(iii) $A^{\dagger}$ is a $h g-k p$.

Proof. Let $A$ and $A^{\dagger}$ be given by (1) and (2), respectively.

(i) $\Rightarrow$ (ii) This follows from $K^{k}=K^{-1} \Leftrightarrow\left(K^{*}\right)^{k}=\left(K^{*}\right)^{-1}$.

(ii) $\Rightarrow$ (i) Since $\left(A^{*}\right)^{*}=A$, the proof follows directly by (i) $\Rightarrow$ (ii) replacing $A$ by $A^{*}$.

(i) $\Rightarrow$ (iii) This follows from $K^{k}=K^{-1} \Leftrightarrow K=\left(K^{-1}\right)^{k}$.

(iii) $\Rightarrow$ (i) Since $\left(A^{\dagger}\right)^{\dagger}=A$, the proof follows directly by (i) $\Rightarrow$ (iii) replacing $A$ by $A^{\dagger}$.

As a corollary we get the part of Theorem 5 in [2].

Corollary $2.7([2])$. Let $A \in \mathbb{C}^{n \times n}$. Then

$$
A \in C_{n}^{H G P} \Leftrightarrow A^{\dagger} \in C_{n}^{H G P} .
$$

The following theorem singles out a sufficient condition for the equivalence of $A$ being a hg-kp and $A$ being an EP matrix.

Theorem 2.8. Let $A \in \mathbb{C}^{n \times n}$. Assume there exists $B \in \mathbb{C}^{n \times n}$ such that $B$ is a hg-kp and $A^{2}=A B$ or $A^{2}=B A$. Then $A$ is a hg-kp if and only if $A \in C_{n}^{E P}$.

Proof. $(\Rightarrow)$ This follows from Theorem 2.1.

$(\Leftarrow)$ Since $B$ is a hg-kp, it is clear that $A^{2}=A B$ leads to

$$
A^{2}=A B B^{\dagger} B=A B^{k+2}=A^{k+3} \text {. }
$$


Now, by using that $A A^{\dagger}=A^{\dagger} A$ and $A^{\dagger} A A^{\dagger}=A^{\dagger}$ and multiplying $A^{2}=A^{k+3}$ three times by $A^{\dagger}$, we have that $A^{\dagger}=A^{k}$. The proof with the condition $A^{2}=B A$ follows similarly.

As the following corollary, we get Theorem 8 in [3].

Corollary 2.9 ([3]). Let $A, B \in \mathbb{C}^{n \times n}$ be such that $B \in C_{n}^{H G P}$ and $A^{2}=A B$ or $A^{2}=B A$. Then $A \in C_{n}^{H G P}$ if and only if $A \in C_{n}^{E P}$.

\section{The Moore-Penrose inverse and the invertibility of a linear combination of commuting hypergeneralized k-projectors}

It is well known that any $\mathrm{g}$-kp is a hg-kp. So, following results also hold for g-kp.

The following lemma is furthermore very useful in this section.

Lemma 3.1. Let $X, Y \in \mathbb{C}^{n \times n}$ and $c_{1}, c_{2} \in \mathbb{C}$. If $X^{k+1}=Y^{k+1}=I_{n}$ and $X Y=Y X$, then

$$
\left(c_{1} X+c_{2} Y\right) \sum_{i=0}^{k}(-1)^{i} c_{1}^{k-i} c_{2}^{i} X^{k-i} Y^{i}=\left(c_{1}^{k+1}+(-1)^{k} c_{2}^{k+1}\right) I_{n} .
$$

Proof. The result follows from

$$
\begin{aligned}
\left(c_{1} X+c_{2} Y\right) \sum_{i=0}^{k}(-1)^{i} c_{1}^{k-i} c_{2}^{i} X^{k-i} Y^{i} & =c_{1}^{k+1} X^{k+1}+(-1)^{k} c_{2}^{k+1} Y^{k+1} \\
& =\left(c_{1}^{k+1}+(-1)^{k} c_{2}^{k+1}\right) I_{n} .
\end{aligned}
$$

In the following theorem, we present the form for the Moore-Penrose inverse and we give some necessary and sufficient conditions for the invertibility of the linear combination $c_{1} A+c_{2} B$, where $A$ and $B$ are two commuting hg-kp.

Theorem 3.1. Let $A \in \mathbb{C}^{n \times n}$ and $B \in \mathbb{C}^{n \times n}$ be commuting hg-kp and let $c_{1}, c_{2} \in \mathbb{C} \backslash\{0\}$ such that $c_{1}^{k+1}+(-1)^{k} c_{2}^{k+1} \neq 0$. Then

$$
\begin{aligned}
\left(c_{1} A+c_{2} B\right)^{\dagger}= & \frac{1}{c_{1}^{k+1}+(-1)^{k} c_{2}^{k+1}}\left(\sum_{i=0}^{k}(-1)^{i} c_{1}^{k-i} c_{2}^{i} A^{k-i} B^{i}\right) A^{k+1} B^{k+1} \\
& +\frac{1}{c_{1}} A^{k}\left(I_{n}-B^{k+1}\right)+\frac{1}{c_{2}} B^{k}\left(I_{n}-A^{k+1}\right) .
\end{aligned}
$$

Furthermore, $c_{1} A+c_{2} B$ is nonsingular if and only if $n=\mathrm{r}(A)+\mathrm{r}(B)-\mathrm{r}(A B)$ and in this case $\left(c_{1} A+c_{2} B\right)^{-1}$ is given by (7).

Proof. By Theorem 2.1 and Corollary 3.9 from [4], we can suppose that $A$ and $B$ have the form

$$
A=U\left(A_{1} \oplus A_{2} \oplus 0_{t} \oplus 0\right) U^{*}, B=U\left(B_{1} \oplus 0_{s} \oplus B_{2} \oplus 0\right) U^{*},
$$


where $U \in \mathbb{C}^{n \times n}$ is unitary, $A_{1}, B_{1} \in \mathbb{C}^{r \times r}, A_{2} \in \mathbb{C}^{s \times s}, B_{2} \in \mathbb{C}^{t \times t}$ are nonsingular, $A_{1} B_{1}=B_{1} A_{1}, A_{1}^{k+1}=B_{1}^{k+1}=I_{r}, A_{2}^{k+1}=I_{s}$ and $B_{2}^{k+1}=I_{t}$. Also, notice that

$$
A^{k+1}=U\left(I_{r} \oplus I_{s} \oplus 0_{t} \oplus 0\right) U^{*}, \quad B^{k+1}=U\left(I_{r} \oplus 0_{s} \oplus I_{t} \oplus 0\right) U^{*} .
$$

Now, we have

$$
c_{1} A+c_{2} B=U\left(\left(c_{1} A_{1}+c_{2} B_{1}\right) \oplus c_{1} A_{2} \oplus c_{2} B_{2} \oplus 0_{n-(r+s+t)}\right) U^{*} .
$$

By Lemma 3.1, we get that $c_{1} A_{1}+c_{2} B_{1}$ is nonsingular and that

$$
\left(c_{1} A_{1}+c_{2} B_{1}\right)^{-1}=\frac{1}{c_{1}^{k+1}+(-1)^{k} c_{2}^{k+1}} \sum_{i=0}^{k}(-1)^{i} c_{1}^{k-i} c_{2}^{i} A_{1}^{k-i} B_{1}^{i} .
$$

Also, $c_{1} A_{2}$ and $c_{2} B_{2}$ are nonsingular and $\left(c_{1} A_{2}\right)^{-1}=\frac{1}{c_{1}} A_{2}^{k}$ and $\left(c_{2} B_{2}\right)^{-1}=$ $\frac{1}{c_{2}} B_{2}^{k}$. Now, we have

$$
\begin{aligned}
& \left(c_{1} A+c_{2} B\right)^{\dagger} \\
= & U\left(\left(\frac{1}{c_{1}^{k+1}+(-1)^{k} c_{2}^{k+1}} \sum_{i=0}^{k}(-1)^{i} c_{1}^{k-i} c_{2}^{i} A_{1}^{k-i} B_{1}^{i}\right) \oplus \frac{1}{c_{1}} A_{2}^{k} \oplus \frac{1}{c_{2}} B_{2}^{k} \oplus 0\right) U^{*} .
\end{aligned}
$$

Finally, using (8), we get (7).

Also, from (9) we can conclude that $c_{1} A+c_{2} B$ is nonsingular if and only if $n=r+t+s$. Since $\mathrm{r}(A)=r+t, \mathrm{r}(B)=r+s$ and $\mathrm{r}(A B)=r$, we get that $c_{1} A+c_{2} B$ is nonsingular if and only if $n=\mathrm{r}(A)+\mathrm{r}(B)-\mathrm{r}(A B)$.

In the following corollaries, we study the problem of when linear combinations $c_{1} I_{n}+c_{2} \prod_{i=1}^{m} A_{i}^{k_{i}}$ and $c_{1} I_{n}+c_{2} A$ are nonsingular, where $\left\{A_{i}\right\}_{i=1}^{m}$ is a finite commuting family of hg-kp and $A$ is a hg-kp, respectively. First, we state the following lemma:

Lemma 3.2. Let $A \in \mathbb{C}^{n \times n}$ and $B \in \mathbb{C}^{n \times n}$ be commuting hg-kp. Then $A B$ is a hg-kp.

Proof. Since $A$ and $B$ are two commuting hg-kp, then $B B^{\dagger} A^{*} A B=A^{*} A B$ and $A B B^{*} A^{\dagger} A=A B B^{*}$, so $(A B)^{\dagger}=A^{\dagger} B^{\dagger}$ (see [10]). Now,

$$
(A B)^{\dagger}=A^{\dagger} B^{\dagger}=A^{k} B^{k}=(A B)^{k} .
$$

Hence, $A B$ is a hg-kp.

Corollary 3.2. Let all of $A_{i} \in \mathbb{C}^{n \times n}, i=\overline{1, m}$ be commuting $h g$ - $k p, m, k_{1}, \ldots$, $k_{m} \in \mathbb{N}, c_{1}, c_{2} \in \mathbb{C}, c_{1} \neq 0$ and $c_{1}^{k+1}+(-1)^{k} c_{2}^{k+1} \neq 0$. Then $c_{1} I_{n}+c_{2} \prod_{i=1}^{m} A_{i}^{k_{i}}$ is nonsingular.

Proof. From Lemma 3.2 we get: if $\left\{A_{i}\right\}_{i=1}^{m}$ is a finite commuting family of hg-kp, then $\prod_{i=1}^{m} A_{i}^{k_{i}}$ is also a hg-kp, where $m, k_{1}, \ldots, k_{m} \in \mathbb{N}$. Now, the proof follows from Theorem 3.1. 
Corollary 3.3. Let $A \in \mathbb{C}^{n \times n}$ be a hg-kp, $c_{1}, c_{2} \in \mathbb{C}, c_{1} \neq 0$ and $c_{1}^{k+1}+$ $(-1)^{k} c_{2}^{k+1} \neq 0$. Then $c_{1} I_{n}+c_{2} A$ is nonsingular and

$\left(c_{1} I_{n}+c_{2} A\right)^{-1}=\frac{1}{c_{1}^{k+1}+(-1)^{k} c_{2}^{k+1}}\left(\sum_{i=0}^{k}(-1)^{i} c_{1}^{k-i} c_{2}^{i} A^{i}\right) A^{k+1}+\frac{1}{c_{1}}\left(I_{n}-A^{k+1}\right)$.

As corollaries we get Theorem 2.1, Proposition 2.3 and Theorem 2.2 in [14], respectively.

Corollary 3.4 ([14]). Let $A \in \mathbb{C}^{n \times n}$ and $B \in \mathbb{C}^{n \times n}$ be commuting g-p or hg-p and let $c_{1}, c_{2} \in \mathbb{C} \backslash\{0\}$ such that $c_{1}^{3}+c_{2}^{3} \neq 0$. Then

$$
\begin{aligned}
\left(c_{1} A+c_{2} B\right)^{\dagger}= & \frac{1}{c_{1}^{3}+c_{2}^{3}}\left(c_{1}^{2} A^{2} B^{3}-c_{1} c_{2} A B+c_{2}^{2} A^{3} B^{2}\right) \\
& +\frac{1}{c_{1}} A^{2}\left(I_{n}-B^{3}\right)+\frac{1}{c_{2}} B^{2}\left(I_{n}-A^{3}\right) .
\end{aligned}
$$

Furthermore, $c_{1} A+c_{2} B$ is nonsingular if and only if $n=\mathrm{r}(A)+\mathrm{r}(B)-\mathrm{r}(A B)$ and in this case $\left(c_{1} A+c_{2} B\right)^{-1}$ is given by (10).

Corollary 3.5 ([14]). Let all of $A_{i} \in \mathbb{C}^{n \times n}, i=\overline{1, m}$ be commuting g-p or $h g-p$, $m, k_{1}, \ldots, k_{m} \in \mathbb{N}, c_{1}, c_{2} \in \mathbb{C}, c_{1} \neq 0$ and $c_{1}^{3}+c_{2}^{3} \neq 0$. Then $c_{1} I_{n}+c_{2} \prod_{i=1}^{m} A_{i}^{k_{i}}$ is nonsingular.

Corollary 3.6 ([14]). Let $A \in \mathbb{C}^{n \times n}$ be a g-p or $h g-p, c_{1}, c_{2} \in \mathbb{C}, c_{1} \neq 0$ and $c_{1}^{3}+c_{2}^{3} \neq 0$. Then $c_{1} I_{n}+c_{2} A$ is nonsingular and

$$
\left(c_{1} I_{n}+c_{2} A\right)^{-1}=\frac{1}{c_{1}^{3}+c_{2}^{3}}\left(c_{1}^{2} A^{3}-c_{1} c_{2} A+c_{2}^{2} A^{2}\right)+\frac{1}{c_{1}}\left(I_{n}-A^{3}\right) .
$$

In the following corollary, we present the form for the Moore-Penrose inverse and we give some necessary and sufficient conditions for the invertibility of linear combination $c_{1} A+c_{2} B$, where $A$ and $B$ are two commuting hg-kp such that $A B=0$.

Corollary 3.7. Let $c_{1}, c_{2} \in \mathbb{C} \backslash\{0\}$. If $A$ and $B$ are commuting hg- $k p$ such that $A B=0$, then

$$
\left(c_{1} A+c_{2} B\right)^{\dagger}=\frac{1}{c_{1}} A^{k}+\frac{1}{c_{2}} B^{k} .
$$

Furthermore, $c_{1} A+c_{2} B$ is nonsingular if and only if $n=\mathrm{r}(A)+\mathrm{r}(B)$ and in this case $\left(c_{1} A+c_{2} B\right)^{-1}$ is given by (11).

If we specialize to $k=2$ in the previous corollary we obtain Corollary 2.4 in [14], which deals with g-p or hg-p.

In the following theorem, we give some necessary and sufficient conditions for the invertibility of linear combination $c_{1} A+c_{2} B+c_{3} C$, where $A, B$ and $C$ are commuting hg-kp such that $B C=0$. 
Theorem 3.8. Let $c_{1}, c_{2}, c_{3} \in \mathbb{C} \backslash\{0\}, c_{1}^{k+1}+(-1)^{k} c_{2}^{k+1} \neq 0$ and $c_{1}^{k+1}+$ $(-1)^{k} c_{3}^{k+1} \neq 0$. If $A, B, C \in \mathbb{C}^{n \times n}$ are commuting hg-kp such that $B C=0$, then the following conditions are equivalent:

(i) $c_{1} A+c_{2} B+c_{3} C$ is nonsingular,

(ii) $B^{k+1}+C^{k+1}+A\left(I_{n}-B^{k+1}-C^{k+1}\right)$ is nonsingular,

(iii) $\mathrm{r}\left(A\left(I_{n}-B^{k+1}-C^{k+1}\right)\right)=n-(\mathrm{r}(B)+\mathrm{r}(C))$.

Proof. By Theorem 2.1 and Corollary 3.9 from [4], we can suppose that $B$ and $C$ have the form

$$
B=U\left(B_{1} \oplus 0_{t} \oplus 0\right) U^{*}, C=U\left(0_{r} \oplus C_{1} \oplus 0\right) U^{*},
$$

where $U \in \mathbb{C}^{n \times n}$ is unitary, $B_{1} \in \mathbb{C}^{r \times r}, C_{1} \in \mathbb{C}^{t \times t}$ are nonsingular, $B_{1}^{k+1}=I_{r}$ and $C_{1}^{k+1}=I_{t}$. Since $A, B, C$ are commuting hg-kp, then $A$ has the form $A=U\left(A_{1} \oplus A_{2} \oplus A_{3}\right) U^{*}$, where $A_{1}, A_{2}, A_{3}$ are hg-kp and $A_{1}$ commutes with $B_{1}$ and $A_{2}$ commutes with $C_{1}$. Now, we get

$$
c_{1} A+c_{2} B+c_{3} C=U\left(\left(c_{1} A_{1}+c_{2} B_{1}\right) \oplus\left(c_{1} A_{2}+c_{3} C_{1}\right) \oplus c_{1} A_{3}\right) U^{*} .
$$

Notice that $c_{1} A_{1}+c_{2} B_{1}$ and $c_{1} A_{2}+c_{3} C_{1}$ are nonsingular. Indeed, since $\left(c_{1} A_{1}\right)^{k+1}+(-1)^{k}\left(c_{2} B_{1}\right)^{k+1}=c_{1}^{k+1} A_{1}^{k+1}+(-1)^{k} c_{2}^{k+1} I_{r}$, then $\left(c_{1} A_{1}\right)^{k+1}+$ $(-1)^{k}\left(c_{2} B_{1}\right)^{k+1}$ is nonsingular because it is the linear combination of an orthogonal projector and the identity matrix for all constants $c_{1}, c_{2} \in \mathbb{C}$ such that $c_{2} \neq 0$ and $c_{1}^{k+1}+(-1)^{k} c_{2}^{k+1} \neq 0$. From nonsingularity of $\left(c_{1} A_{1}\right)^{k+1}+$ $(-1)^{k}\left(c_{2} B_{1}\right)^{k+1}$ and Lemma 3.1, it follows that $c_{1} A_{1}+c_{2} B_{1}$ is nonsingular. Similarly, we can conclude that $c_{1} A_{2}+c_{3} C_{1}$ is nonsingular for all constants $c_{1}, c_{3} \in \mathbb{C}$ such that $c_{3} \neq 0$ and $c_{1}^{k+1}+(-1)^{k} c_{3}^{k+1} \neq 0$. Now, it follows that $c_{1} A+c_{2} B+c_{3} C$ is nonsingular if and only if $A_{3}$ is nonsingular for all constants $c_{1}, c_{2}, c_{3} \in \mathbb{C} \backslash\{0\}$ such that $c_{1}^{k+1}+(-1)^{k} c_{2}^{k+1} \neq 0$ and $c_{1}^{k+1}+(-1)^{k} c_{3}^{k+1} \neq 0$ and we can prove that (i) $\Leftrightarrow\left(\right.$ ii) and (i) $\Leftrightarrow\left(\right.$ iii). Indeed, $A_{3}$ is nonsingular if and only if $B^{k+1}+C^{k+1}+A\left(I_{n}-B^{k+1}-C^{k+1}\right)$ is nonsingular, so (i) $\Leftrightarrow$ (ii). On the other hand, $A_{3}$ is nonsingular if and only if $\operatorname{r}\left(A_{3}\right)=n-(r+t)$, i.e., $\mathrm{r}\left(A\left(I_{n}-B^{k+1}-C^{k+1}\right)\right)=n-(\mathrm{r}(B)+\mathrm{r}(C))$. So (i) $\Leftrightarrow($ iii $)$.

Note that Theorem 2.10 in [14] which deals with g-p or hg-p is the corollary of the previous theorem.

In the following corollary, we study the problem of when a linear combination $c_{1} I_{n}+c_{2} A+c_{3} B$ of commuting hg-kp is nonsingular and we give the form of the inverse in this case.

Corollary 3.9. Let $c_{1}, c_{2}, c_{3} \in \mathbb{C}, c_{1} \neq 0, c_{1}^{k+1}+(-1)^{k} c_{2}^{k+1} \neq 0$ and $c_{1}^{k+1}+$ $(-1)^{k} c_{3}^{k+1} \neq 0$. If $A, B \in \mathbb{C}^{n \times n}$ are commuting hg-kp such that $A B=0$, then $c_{1} I_{n}+c_{2} A+c_{3} B$ is nonsingular and

$$
\left(c_{1} I_{n}+c_{2} A+c_{3} B\right)^{-1}=\frac{1}{c_{1}^{k+1}+(-1)^{k} c_{2}^{k+1}}\left(\sum_{i=0}^{k}(-1)^{i} c_{1}^{k-i} c_{2}^{i} A^{i}\right) A^{k+1}
$$




$$
\begin{aligned}
& +\frac{1}{c_{1}^{k+1}+(-1)^{k} c_{3}^{k+1}}\left(\sum_{i=0}^{k}(-1)^{i} c_{1}^{k-i} c_{3}^{i} B^{i}\right) B^{k+1} \\
& +\frac{1}{c_{1}}\left(I_{n}-A^{k+1}-B^{k+1}\right) .
\end{aligned}
$$

Proof. The invertibility of $c_{1} I_{n}+c_{2} A+c_{3} B$ follows from Theorem 3.8. Also, from the proof of Theorem 3.8 follows that $c_{1} I_{n}+c_{2} A+c_{3} B$ has the form

$$
c_{1} I_{n}+c_{2} A+c_{3} B=U\left(\left(c_{1} I_{r}+c_{2} A_{1}\right) \oplus\left(c_{1} I_{t}+c_{3} B_{1}\right) \oplus c_{1} I_{n-r-t}\right) U^{*},
$$

where $U \in \mathbb{C}^{n \times n}$ is unitary, $A_{1} \in \mathbb{C}^{r \times r}, B_{1} \in \mathbb{C}^{t \times t}$ are nonsingular, $A_{1} B_{1}=$ $B_{1} A_{1}, A_{1}^{k+1}=I_{r}$ and $B_{1}^{k+1}=I_{t}$. By Corollary 3.3 , we get

$$
\left(c_{1} I_{r}+c_{2} A_{1}\right)^{-1}=\frac{1}{c_{1}^{k+1}+(-1)^{k} c_{2}^{k+1}}\left(\sum_{i=0}^{k}(-1)^{i} c_{1}^{k-i} c_{2}^{i} A_{1}^{i}\right) A_{1}^{k+1}
$$

and

$$
\left(c_{1} I_{t}+c_{3} B_{1}\right)^{-1}=\frac{1}{c_{1}^{k+1}+(-1)^{k} c_{3}^{k+1}}\left(\sum_{i=0}^{k}(-1)^{i} c_{1}^{k-i} c_{3}^{i} B_{1}^{i}\right) B_{1}^{k+1} .
$$

Now,

$$
\begin{aligned}
\left(c_{1} I_{n}+c_{2} A+c_{3} B\right)^{-1}= & U\left(\frac{1}{c_{1}^{k+1}+(-1)^{k} c_{2}^{k+1}}\left(\sum_{i=0}^{k}(-1)^{i} c_{1}^{k-i} c_{2}^{i} A_{1}^{i}\right) A_{1}^{k+1}\right. \\
& \oplus \frac{1}{c_{1}^{k+1}+(-1)^{k} c_{3}^{k+1}}\left(\sum_{i=0}^{k}(-1)^{i} c_{1}^{k-i} c_{3}^{i} B_{1}^{i}\right) B_{1}^{k+1} \\
& \left.\oplus \frac{1}{c_{1}} I_{n-r-t}\right) U^{*}
\end{aligned}
$$

which is equivalent to (12).

If we specialize to $k=2$ in the previous corollary we obtain Theorem 2.11 in [14], which deals with g-p or hg-p.

Acknowledgement. The author would like to thank the anonymous reviewers for their very useful comments that helped to improve the presentation of this paper.

\section{References}

[1] O. M. Baksalary, Revisitation of generalized and hypergeneralized projectors, Statistical Inference, Econometric Analysis and Matrix Algebra VI (2009), 317-324.

[2] J. K. Baksalary, O. M. Baksalary, and X. Liu, Further properties of generalized and hypergeneralized projectors, Linear Algebra Appl. 389 (2004), 295-303.

[3] J. K. Baksalary, O. M. Baksalary, X. Liu, and G. Trenkler, Further results on generalized and hypergeneralized projectors, Linear Algebra Appl. 429 (2008), no. 5-6, 1038-1050.

[4] J. Benítez, Moore-Penrose inverses and commuting elements of $C^{*}$-algebras, J. Math. Anal. Appl. 345 (2008), no. 2, 766-770. 
[5] J. Benítez and N. Thome, Characterizations and linear combinations of k-generalized projectors, Linear Algebra Appl. 410 (2005), 150-159.

$[6] \longrightarrow,\{k\}$-group periodic matrices, SIAM. J. Matrix Anal. Appl. 28 (2006), no. 1, $9-25$.

[7] A. Berman, Nonnegative matrices which are equal to their generalized inverse, Linear Algebra Appl. 9 (1974), 261-265.

[8] S. L. Campbell and C. D. Meyer Jr., Generalized Inverses of Linear Transformations, Pitman, London, 1979.

[9] C. Y. Deng, Q. H. Li, and H. K. Du, Generalized n-idempotents and Hyper-generalized $n$-idempotents, Northeast. Math. J. 22 (2006), no. 4, 387-394.

[10] T. N. E. Greville, Note on the generalized inverse of a matrix product, SIAM Rev. 8 (1966), 518-521.

[11] J. Groß and G. Trenkler, Generalized and hypergeneralized projectors, Linear Algebra Appl. 264 (1997), 463-474.

[12] R. E. Hartwig and K. Spindelböck, Matrices for which $A^{*}$ and $A^{\dagger}$ commute, Linear Multilinear Algebra 14 (1983), no. 3, 241-256.

[13] M. Tošić and D. S. Cvetković-Ilić, The invertibility of the difference and the sum of commuting generalized and hypergeneralized projectors, Linear Multilinear Algebra 61 (2013), no. 4, 482-493.

[14] M. Tošić, D. S. Cvetković-Ilić, and C. Deng, The Moore-Penrose inverse of a linear combination of commuting generalized and hypergeneralized projectors, Electron. J. Linear Algebra 22 (2011), 1129-1137.

Faculty of Sciences and Mathematics

UNIVERSITY OF NIŠ

SERBIA

E-mail address: marinatosic@ymail.com 